\title{
A Survey of Tick-Borne Bacterial Pathogens in Florida
}

\author{
Carrie E. De Jesus ${ }^{1}$, Claudia Ganser ${ }^{2}$, William H. Kessler ${ }^{2}{ }^{\mathbb{D}}$, Zoe S. White ${ }^{1}$, \\ Chanakya R. Bhosale ${ }^{3}$, Gregory E. Glass ${ }^{2}$ and Samantha M. Wisely ${ }^{1, *}$ (i) \\ 1 Department of Wildlife Ecology and Conservation, University of Florida, Gainesville, FL 32611, USA; \\ carriedejesus@ufl.edu (C.E.D.J.); zseganish@ufl.edu (Z.S.W.) \\ 2 Department of Geography, University of Florida, Gainesville, FL 32611, USA; gancla@ufl.edu (C.G.); \\ wkessler@ufl.edu (W.H.K.); gglass@epi.ufl.edu (G.E.G.) \\ 3 Department of Entomology and Nematology, University of Florida, Gainesville, FL 32611, USA; \\ cbhosale@ufl.edu \\ * Correspondence: wisely@ufl.edu
}

Received: 25 June 2019; Accepted: 11 September 2019; Published: 13 September 2019

\begin{abstract}
Within the past three decades, new bacterial etiological agents of tick-borne disease have been discovered in the southeastern U.S., and the number of reported tick-borne pathogen infections has increased. In Florida, few systematic studies have been conducted to determine the presence of tick-borne bacterial pathogens. This investigation examined the distribution and presence of tick-borne bacterial pathogens in Florida. Ticks were collected by flagging at 41 field sites, spanning the climatic regions of mainland Florida. DNA was extracted individually from 1608 ticks and screened for Anaplasma, Borrelia, Ehrlichia and Rickettsia using conventional PCR and primers that amplified multiple species for each genus. PCR positive samples were Sanger sequenced. Four species of ticks were collected: Amblyomma americanum, Amblyomma maculatum, Dermacentor variabilis, and Ixodes scapularis. Within these ticks, six bacterial species were identified: Borrelia burgdorferi, Borrelia lonestari, Ehrlichia ewingii, Rickettsia amblyommatis, Rickettsia andeanae, Rickettsia parkeri, and Rickettsia endosymbionts. Pathogenic Borrelia, Ehrlichia, and Rickettsia species were all detected in the North and North-Central Florida counties; however, we found only moderate concordance between the distribution of ticks infected with pathogenic bacteria and human cases of tick-borne diseases in Florida. Given the diversity and numerous bacterial species detected in ticks in Florida, further investigations should be conducted to identify regional hotspots of tick-borne pathogens.
\end{abstract}

Keywords: tick-borne disease; Florida; surveillance; Rickettsia; Borrelia; Ehrlichia; Amblyomma americanum

\section{Introduction}

The number of reported tick-borne pathogen infections has more than doubled in the United States over the past decade according to the U.S. Center of Disease Control and Prevention (CDC) [1] In response, state and federal surveillance efforts have increased, and the majority of those efforts have been concentrated in the Northeastern and Upper Midwest of the U.S. to understand epidemiology and disease ecology of Borrelia burgdorferi, the causative agent of Lyme disease. However, in the Southeastern U.S. the number of tick-borne bacterial infections causing ehrlichiosis, spotted fever rickettsiosis and Lyme disease is increasing [2,3]. Surveillance in this region has detected multiple etiological agents (Borrelia, Rickettsia, Ehrlichia, and Anaplasma) that cause human and animal disease [2-7]. Within the past three decades, a diverse array of new bacterial etiological agents of tick-borne disease have been discovered in the U.S. including E. chaffensis, E. ewingii, R. parkeri, and Panola Mountain Ehrlichia sp. (PME) [8-11]. Given the discovery and diversity of tick-borne bacterial pathogens detected in the U.S., there is a substantial need for ongoing pathogen surveillance of ticks nationally. 
To address the need for pathogen surveillance, the CDC has developed standardized protocols to survey for tick species and pathogens [12] in order to monitor changes in the distribution and abundance of ticks, as well as to detect the presence and estimate the prevalence of tick-borne pathogens. These data can be used as part of a larger program that addresses the questions of when and where people may be exposed to ticks and their associated pathogens [12,13]. The specific objective of the surveillance protocol is to determine the presence of ticks and their associated pathogens at the appropriate spatial scale throughout the United States [12].

Florida has been a largely neglected state in the surveillance of tick-borne bacterial pathogens. Florida is divided into seven distinct climatic regions that vary from north (temperate) to south (subtropical), making it more climatically diverse than other southeastern states (Figure 1) [14]. The variation between temperate to subtropical climate could influence the distribution of ticks and bacterial pathogens present [14,15]. Previous studies of ticks and bacterial pathogens have been restricted geographically from the Panhandle to Central Florida (regions 1-3), which are climatically similar to other southeastern states $[6,13,15-18]$. Florida has multiple tick species that are vectors for bacterial pathogens: Amblyomma americanum, A. maculatum, Dermacentor variabilis, Ixodes scapularis and Rhipicephalus sanguineus [15-18].

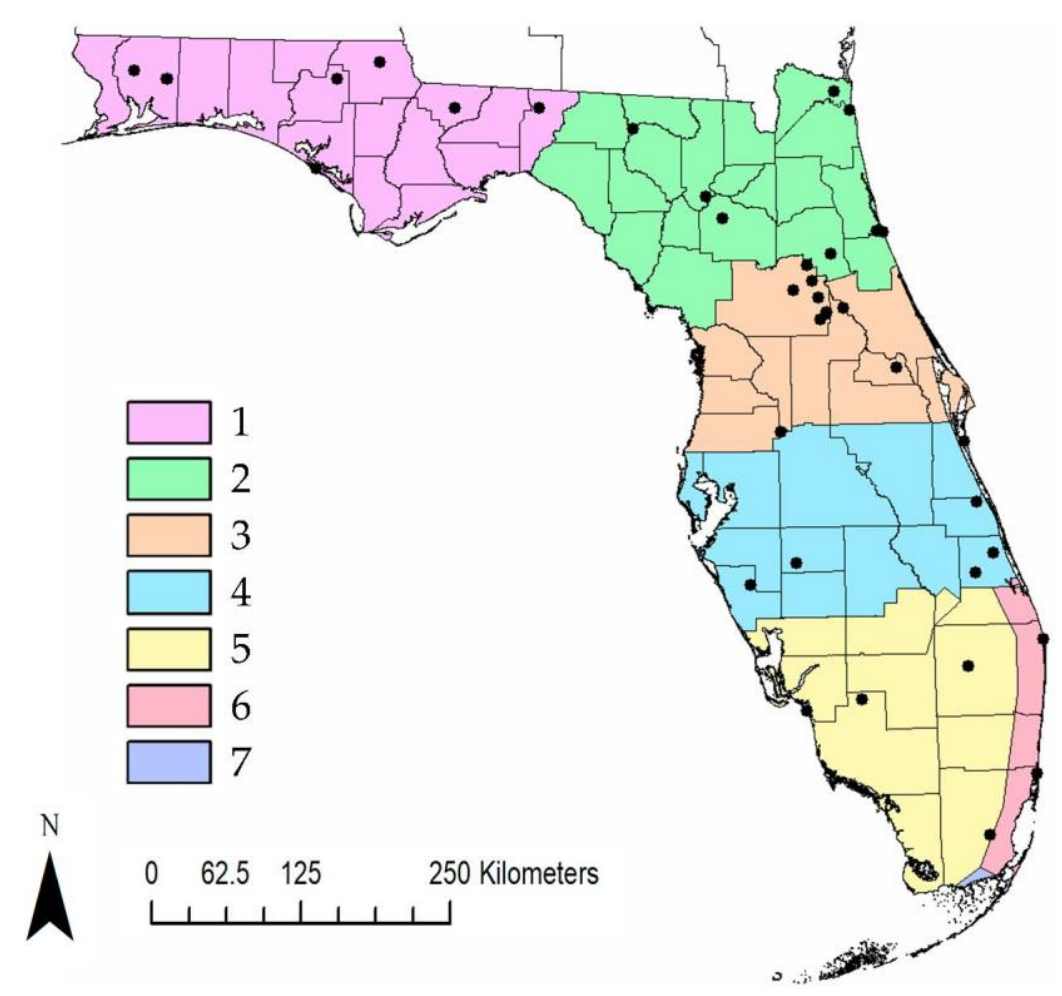

Figure 1. Map of the seven climatic regions of Florida. Each color represents a climatic region going from north to south. Black dots indicate field sites where ticks were collected.

Within the last eight years, human cases of tick-borne disease have been reported in 42 of the 67 counties in Florida with $>50$ cases per year acquired in state (Table 1) [19]. The primary tick-borne illnesses of concern in Florida are Lyme disease, ehrlichiosis and spotted fever rickettsiosis. Cases of tick-borne diseases in Florida occur between the months of April through August. Lyme disease is the most commonly reported tick-borne disease; however, the majority of reported cases are reported to be acquired out of state. Previous studies in Florida have detected B. burgdorferi sensu stricto in I. scapularis adults and detected Borrelia lonestari, a bacterium of unknown pathogenicity, in A. americanum [20,21]. 
Table 1. Acquired cases of tick-borne diseases in Florida. Includes confirmed and probable cases (Florida Department of Health, Morbidity and Mortality Report 2011-2017).

\begin{tabular}{cccccccc}
\hline Disease & $\mathbf{2 0 1 1}$ & $\mathbf{2 0 1 2}$ & $\mathbf{2 0 1 3}$ & $\mathbf{2 0 1 4}$ & $\mathbf{2 0 1 5}$ & $\mathbf{2 0 1 6}$ & $\mathbf{2 0 1 7}$ \\
\hline Anaplasmosis & 6 & 1 & 0 & 0 & 0 & 1 & 0 \\
Ehrlichiosis & 13 & 20 & 17 & 23 & 16 & 23 & 12 \\
Lyme disease & 22 & 39 & 21 & 35 & 35 & 36 & 27 \\
$\begin{array}{c}\text { Spotted fever } \\
\text { rickettsiosis }\end{array}$ & 9 & 20 & 15 & 21 & 12 & 7 & 15 \\
\hline
\end{tabular}

Ehrlichiosis is a locally prevalent disease in Florida. Multiple vertebrate species (white-tailed deer (Odocoileus virginianus), raccoons (Procyon lotor), wild pigs (Sus scrofa) and opossums (Didelphis virginiana) have been found to be seropositive for Ehrlichia species in Florida [8,18,22-24]. Previous studies have reported infected $A$. americanum throughout the central part of the state for both Ehrlichia ewingii and E. chaffeensis $[17,18]$.

In Florida approximately a dozen cases of spotted fever rickettsiosis are reported each year; however, ticks are commonly infected with a variety of Rickettsia species that can be either endosymbionts or pathogenic to humans and animals. Rickettsia species that cause spotted fever rickettsiosis can induce mild to severe symptoms. Rickettsia rickettsii, the pathogen of Rocky Mountain spotted fever (RMSF), is the most severe of the Rickettsioses, which can result in fatality if untreated. This pathogen is mainly vectored by $D$. variabilis, but $A$. americanum and $R$. sanguineus are also considered vector species [25-28]. Infections of $R$. rickettsii by $R$. sanguineus have been identified in communities with peridomestic dogs heavily infested with ticks, including in Florida $[27,28]$. In contrast $R$. parkeri is less severe and can result in mild fever and eschar [11]. Rickettsia parkeri is primarily vectored by A. maculatum but has also been found in A. americanum [28]. Rickettsia amblyommatis has been reported as a possibly pathogenic Rickettsia that produces milder symptoms than $R$. parkeri or $R$. rickettsii and is the most common bacterial pathogen reported in A. americanum $[4,6,28,29]$. Nationwide, the number of spotted fever cases has increased, yet this trend is accompanied by a decrease in case fatality, suggesting that an increase in the prevalence of less pathogenic species like R. amblyommatis is the cause of the trend in human cases [30].

Florida has multiple tick species that are capable of vectoring a variety of tick-borne bacterial pathogens. Given the limited geographic scope of previous tick-borne pathogen studies yet broad geographic scope of human cases, further investigation is needed to better understand the distribution of tick-borne bacterial pathogens across the entire state. The purpose of our investigation was to examine the distribution of tick-borne bacterial pathogens within the six climatic regions of the Florida mainland. Our survey followed the guidelines of the CDC protocol as implemented by Glass et al. [31] for the detection of pathogens among distinct climatic regions. Our study discusses results in the context of the presence of pathogens and human acquired cases of tick-borne diseases to provide a more comprehensive picture of tick-associated pathogens and human exposure [13].

\section{Materials and Methods}

\subsection{Tick Collections}

We collected ticks at field sites across the 6 climatic regions of mainland Florida using the methodology of Glass et al. [31] and Kessler et al., 2018 [32]. Briefly, sampling effort was stratified across the range of climatic regions that varied across the latitudinal gradient of Florida, included 41 field sites, and incorporated 28 of the 67 Florida counties following CDC guidelines for collecting tick pathogen data (Figure 1) [12]. We collected ticks by flagging $\left(1 \mathrm{~m}^{2}\right.$, felt $)$ on transects of 100-200 m 4-6 times a year. Ticks collected from 2016 through August 2017 were stored in 70\% ethanol. Collection protocols changed in September 2017, and all samples through 2018 were stored in 100\% ethanol and kept cool until they could be stored at $-80^{\circ} \mathrm{C}$, where they were stored until processing. All ticks were identified by sex, life stage, and species using taxonomic keys [33-36]. Seasonality was also examined for each tick species collected. 


\subsection{DNA Extractions}

We extracted only DNA from ticks collected from 2016 through August 2017. To extract DNA, adult and nymphal ticks were cut in half on a piece of parafilm using flame-sterilized forceps and scalpel. Half of each tick was then placed into proteinase K digestion and ATL buffer and digested overnight at $56{ }^{\circ} \mathrm{C}$ (QIAGEN, Valencia, CA, USA). When single larvae were found, they were directly placed into the proteinase K digestion. Once digested, the Qiagen DNeasy extraction kit (QIAGEN, Valencia, CA, USA) was used according to the manufacturer's protocol [37]. The quality and quantity for each tick measured on a Nanodrop (Nanodrop One, Thermo Fisher Scientific, Carlsbad, CA, USA). Quality of DNA was also visualized on a gel in order to check for high molecular weight. In order to prevent contamination, DNA and RNA extractions were conducted in a separate room from the PCR preparation and products. The extracted DNA was stored at $-20^{\circ} \mathrm{C}$ and RNA at $-80^{\circ} \mathrm{C}$ until PCR protocols were implemented.

From September 2017 through October 2018, ticks were extracted using the Trizol reagent (Thermo Fisher Scientific, Carlsbad, CA, USA) in order to extract both RNA and DNA. Adult ticks were cut in half on a piece of parafilm before being placed into the Trizol buffer using a flame-sterilized and RNase-free scalpel. Nymphal ticks were cut on the bottom quarter of the tick, and singleton larvae were directly placed into Trizol. Once in Trizol, all ticks were sonicated. Adults were sonicated with 3 pulses but nymphs and larvae were processed with 2 pulses, each pulse was $5 \mathrm{~s}$ each at $25 \%$ amplitude (Q125 QSonica Sonicator, Newtown, CT, USA). The rest of the Trizol protocol was followed according to manufacturer instructions [38]. Once DNA and RNA were extracted, DNA was stored at $-20^{\circ} \mathrm{C}$ and RNA at $-80^{\circ} \mathrm{C}$ until PCR protocols were run.

Because we changed DNA extraction protocols, we compared both methods to determine if there were any differences in quality and quantity of the DNA using unpaired $t$-tests. In addition, we conducted a logit regression to determine if the quantity of DNA had an impact on our ability to amplify bacterial DNA. All statistical analyses were conducted using the base package in R [39].

\subsection{Pathogen Screening}

Our pathogen screening methods allowed for the detection of a wide array of tick-borne bacterial pathogens of concern to human health by using primers that detected multiple species within a genus of bacteria. Ticks were screened for Anaplasma, Ehrlichia, Borrelia, and Rickettsia species. For Ehrlichia and Anaplasma the PCR targeted the groESL gene, Borrelia targeted the Flagellin b gene, and Rickettsia the ompA gene (Table 2) [40-42]. Individual adults, nymphs, and larvae were tested for each bacterial pathogen.

Table 2. Published PCR assays used in this survey. The table includes the targeted gene, primer sequence and positive controls.

\begin{tabular}{|c|c|c|c|c|}
\hline Pathogen & Target Gene & Primer Sequences 5'-3' & Positive Control & Reference \\
\hline Anaplasma/Ehrlichia & groESL & $\begin{array}{c}\text { gro607F } \\
\text { GAAGATGC(A/T)GT(A/T)GG(A/T)TGTAC(G/T)GC } \\
\text { gro677F } \\
\text { ATTACTCAGAGTGCTTCTCA(A/G)TG } \\
\text { gro1294R } \\
\text { AG(A/C)GCTTC(A/T)CCTTC(A/T)AC(A/G)TC(C/T)TC } \\
\text { gro1121R } \\
\text { TGCATACC(A/G)TCAGT(C/T)TTTTCAAC }\end{array}$ & E. chaffensis & [40] \\
\hline Borrelia & Flagellin $b$ & $\begin{array}{c}\text { FlaLL } \\
\text { FlaRL } \\
\text { ACATATTCAGATGCAGACAGA } \\
\text { GCAATCATAGCCATTGCAGATTGT } \\
\text { FlaLS } \\
\text { AACAGCTGAAGAGCTTGGAATG } \\
\text { FlaRS } \\
\text { CTTTGATCACTTATCATTCTAATAGC }\end{array}$ & B. lonestari & [41] \\
\hline Rickettsia & ompA & $\begin{array}{c}\mathbf{R r} \mathbf{1 9 0 . 7 0 p} \\
\text { ATGGCGAATATTTCTCCAAAA } \\
190.701 \\
\text { GTTCCGTTAATGGCAGCATCT }\end{array}$ & R.sp endosymbiont & [42] \\
\hline
\end{tabular}


PCR amplification protocols were conducted on a Master cycler pro S (Eppendorf, Hamburg, Germany). Positive controls used in the PCR protocols were derived from DNA extractions from an A. americanum that was positive for E. chaffeensis and B. lonestari. For the Rickettsia positive control, DNA from a Rickettsia spp. endosymbiont was extracted from an I. scapularis. Lastly, a supernatant of infected dog cells was extracted for the positive control of A. phagocytophilum. A negative control was also run for each PCR assay with PCR grade water. PCR products were run on a gel using RedView DNA Gel Stain ${ }^{\mathrm{TM}}$ (Genecopoeia, Rockville, MD, USA) to determine if the gene sequences of interest were amplified. Samples were considered positive for a bacterium if a band for the appropriate sequence size was present on a gel. All samples that tested positive for a pathogen were sent to GenScript (Piscataway, NJ, USA) for Sanger sequencing. Sequences were aligned using Geneious (v 11.1) and then compared to sequences in GenBank using NCBI BLAST. All pathogen sequences matched sequences available in the NCBI database. Genetic sequences for each bacterial species were submitted to NCBI.

\section{Results}

\subsection{Tick Collections}

In total 1608 ticks were collected from March 2016 through October 2018 (Table 3). Ticks were collected from all six climatic regions and 20/28 counties surveyed by flagging. Of the ticks collected, A. americanum $(n=1325)$ was the most abundant and was found in $16 / 28$ counties (Table 3 ). In addition, 233 I. scapularis were collected in $14 / 28$ counties and 46 D. variabilis in 4/28 counties (Table 3 ). Lastly, A. maculatum was the least abundant tick, with only two nymphs and two adults collected in 4/28 counties. Amblyomma and Dermacentor ticks were abundant during the spring and summer months, while Ixodes adults were more abundant during the winter months.

Table 3. Species identity and sample size of ticks screened across the entire state of Florida between spring 2016 and fall 2018.

\begin{tabular}{|c|c|c|c|c|c|c|c|c|c|c|c|c|}
\hline Species & $\begin{array}{c}\text { Spring } \\
16\end{array}$ & $\begin{array}{c}\text { Summer } \\
16\end{array}$ & $\begin{array}{c}\text { Fall } \\
16\end{array}$ & $\begin{array}{c}\text { Winter } \\
16-17\end{array}$ & $\begin{array}{c}\text { Spring } \\
17\end{array}$ & $\begin{array}{c}\text { Summer } \\
17\end{array}$ & $\begin{array}{c}\text { Fall } \\
17\end{array}$ & $\begin{array}{l}\text { Winter } \\
17-18\end{array}$ & $\begin{array}{c}\text { Spring } \\
18\end{array}$ & $\begin{array}{c}\text { Summer } \\
18\end{array}$ & $\begin{array}{c}\text { Fall } \\
18\end{array}$ & Total \\
\hline \multicolumn{13}{|c|}{ A. americanum } \\
\hline Adult & 60 & 45 & 0 & 0 & 52 & 0 & 5 & 34 & 33 & 173 & 0 & 402 \\
\hline Nymph & 133 & 89 & 0 & 8 & 75 & 0 & 75 & 30 & 238 & 106 & 156 & 910 \\
\hline Larvae & 0 & 2 & 0 & 2 & 0 & 0 & 1 & 6 & 2 & 0 & 0 & 13 \\
\hline \multicolumn{13}{|c|}{ A. maculatum } \\
\hline Adult & 0 & 0 & 0 & 0 & 0 & 0 & 1 & 0 & 0 & 1 & 0 & 2 \\
\hline Nymph & 0 & 0 & 0 & 0 & 1 & 0 & 0 & 1 & 0 & 0 & 0 & 2 \\
\hline \multicolumn{13}{|c|}{ D. variabilis } \\
\hline Adult & 6 & 8 & 0 & 0 & 5 & 0 & 2 & 1 & 10 & 8 & 6 & 46 \\
\hline \multicolumn{13}{|l|}{ I. scapularis } \\
\hline Adult & 10 & 1 & 0 & 94 & 62 & 0 & 7 & 18 & 36 & 2 & 0 & 230 \\
\hline Nymph & 2 & 0 & 0 & 1 & 0 & 0 & 0 & 0 & 0 & 0 & 0 & 3 \\
\hline
\end{tabular}

\subsection{Pathogen Screening}

We found ticks that tested positive for Borrelia, Ehrlichia, and Rickettsia species, but no Anaplasma species were detected (Figure 2, Table 4). Two species of Borrelia were detected; B. burgdorferi and B. lonestari. B. burgdorferi was detected in one adult $I$. scapularis and B. lonestari in 16 A. americanum and one D. variabilis. For Ehrlichia only one species was detected, E. ewingii. Two A. americanum tested positive for E. ewingii. The two ticks infected with E. ewingii were also co-infected with $R$. amblyommatis. $R$. amblyommatis was the most abundant bacteria found in ticks with 410 infected A. americanum detected. $R$. amblyommatis had the highest prevalence compared to all other pathogen species detected and was most prevalent in ticks collected from the spring and summer months (Figure 3). Two A. americanum larvae were positive for R. parkeri. R. andeanae was detected in 1 A. maculatum. Lastly, I. scapularis and $D$. variabilis were both found to be infected with Rickettsia endosymbionts. 
A Borrelia burgdorferi

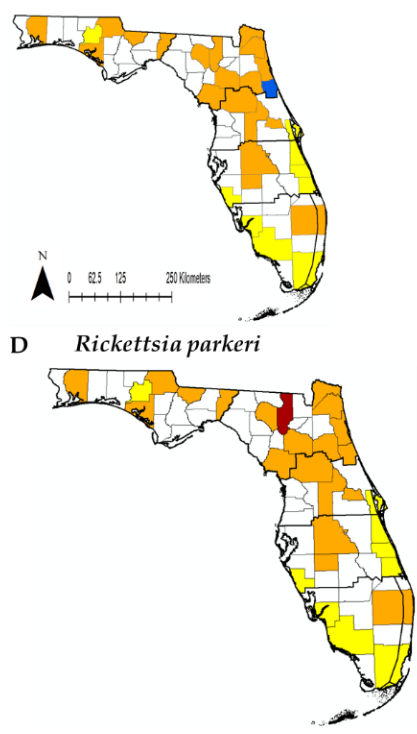

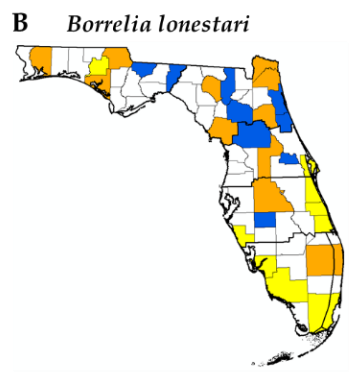

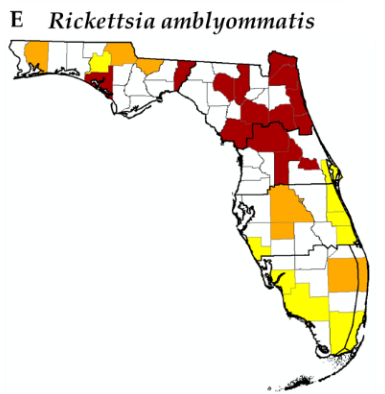

C Ehrlichia ewingii

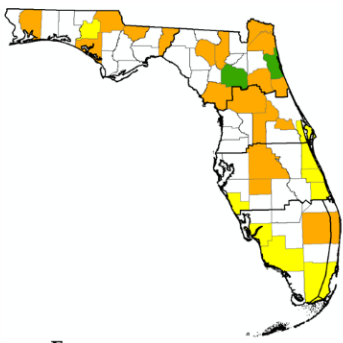

F Rickettsia andeanae

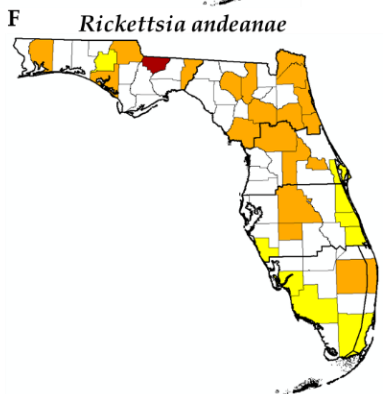

Figure 2. Florida counties where ticks were surveyed, and ticks that were positive for a bacterial species. Yellow $=$ site surveyed but ticks not collected, Orange $=$ counties where ticks were collected but were not infected with a bacterial species. Red/blue/green = county with positive ticks for a genus of bacterial species detected. Ixodes scapularis vectors: Thick black lines denote climatic regions. (A) B. burgdorferi. Amblyomma americanum vectors: (B) B. lonestari, (C) E. ewingii, (D) R. parkeri, (E) R. amblyommatis. Amblyomma maculatum vectors: (F) R. andeanae.

Table 4. Pathogen prevalence of ticks collected from Spring 2016 through Fall 2018. * Most commonly reported BLAST result for each pathogen.

\begin{tabular}{cccc}
\hline Tick Species & Pathogen Species & $\begin{array}{c}\text { No. Positive/No. Tested } \\
\mathbf{( \% )}[\mathbf{9 5 \%} \mathbf{C I}]\end{array}$ & \% Identical (Accession \#) * \\
\hline I. scapularis & B. burgdorferi & $1 / 233(0.43 \%)[0-0.02]$ & $98 \%(\mathrm{AF} 264895.1)$ \\
I. scapularis & R. endosymbiont & $80 / 233(34.3 \%)[0.3-0.42]$ & $98 \%(\mathrm{AB} 002268)$ \\
D. variabilis & R. endosymbiont & $1 / 46(2.2 \%)[0-0.11]$ & $97 \%(\mathrm{AB} 002268)$ \\
D. variabilis & B. lonestari & $1 / 46(2.2 \%)[0-0.11]$ & $99 \%(\mathrm{AF} 273670)$ \\
A. americanum & B. lonestari & $17 / 1312(1.29 \%)$ & $100 \%(\mathrm{AF} 273670)$ \\
A. americanum & E. ewingii & $2 / 1312(0.16 \%)[0-0.001]$ & $98 \%(\mathrm{AF} 195273)$ \\
A. americanum & R. amblyommatis & $391 / 1312(29 \%)$ & $100 \%(\mathrm{CP} 003334)$ \\
A. americanum & R. parkeri & $2 / 1312(0.16 \%)[0-0.01]$ & $99.7 \%(\mathrm{MH} 247927)$ \\
A. maculatum & R. andeanae & $1 / 4(25 \%)[0.05-0.7]$ & $100 \%(\mathrm{KY} 628370)$ \\
\hline
\end{tabular}

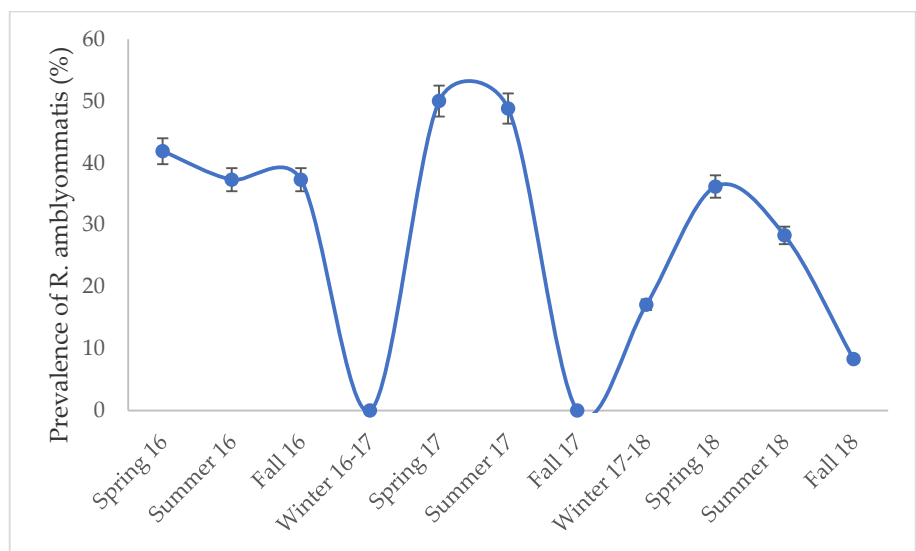

Figure 3. Prevalence $\pm 95 \%$ confidence interval (CI) of R. amblyommatis in all life stages of A. americanum over a three-year collection period. Amblyomma americanum were not collected in winter 1-17 and fall 17. 


\subsection{Comparison of DNA Extraction Protocols}

DNA concentrations used in PCR ranged from 1.91-158.4 ng for Qiagen and 2.5-420.8 ng for Trizol protocols. For the Qiagen protocol the average DNA concentration for adults, nymphs and larvae were 37.87 (95\% CI, \pm 2.82$), 18.22( \pm 2.14)$, and $20.05 \mathrm{ng} / \mu \mathrm{l}( \pm 14.74)$. The Trizol protocol averages for DNA concentrations were $92.15( \pm 4.83), 130.36( \pm 6.72)$, and $199.80 \mathrm{ng} / \mu \mathrm{L}( \pm 73.93)$. The Trizol extracted significantly more DNA compared to the Qiagen protocol $(t$-test, $t=34.4, \mathrm{df}=1412.3, p<0.0001)$. We had an average 280/260 value of $1.67( \pm 0.22)$ for the Trizol protocol and $1.79( \pm 0.02)$ for Qiagen. We did see significant difference between the $280 / 260(t=3.317, \mathrm{df}=1103.2, p=0.001)$. For the $260 / 230$ values we obtained an average value of $1.34( \pm 0.19)$ for the Trizol and $1.31( \pm 0.18)$ for the Qiagen protocol. We did not see significant differences in the 260/230 values between both extraction protocols $(t=1.3545$, $\mathrm{df}=1637.9, p$-value $=0.1758)$. DNA quantity of DNA did not impact our PCR results for the Trizol (logit, $\mathrm{df}=1041, \mathrm{z}=-0.073, p=0.941$ ) and Qiagen protocols (logit, $\mathrm{df}=686, z=0.153, p=0.878$ ).

\subsection{Pathogen Detection and Human Cases in Florida}

Borrelia, Ehrlichia and Rickettsia were all detected within climatic regions 1-3. (Figure 3). Borrelia burgdorferi was only detected in one county within climatic region 2 (Figure 3) even though human cases for locally acquired B.burgdorferi infection were spread throughout the entire state (Figure 4). Borrelia lonestari was detected across climatic regions 1-3 and the only pathogen detected in region 4 . Ehrlichia ewingii was found in climatic region 2. Our pathogen survelliance for Ehrlichia did not overlap with human cases at the county level. Human cases of Ehrlichiosis did overlap with climatic regions 2-3 and with a few cases in regions 4-6. Human cases of rickettsiosis were concentrated in climatic regions 1-3, with a few cases in region 4 . Rickettsia-postive ticks were found throughout cimatic regions 1-3. Ticks infected with Rickettsia overlapped with reported human cases for spotted fever rickettsiosis at the county level within climatic region 2 (Figure 4). Ticks were collected in climatic region 4 but they were not infected with Rickettsia, even though human cases of rickettsiosis have been reported in the region.

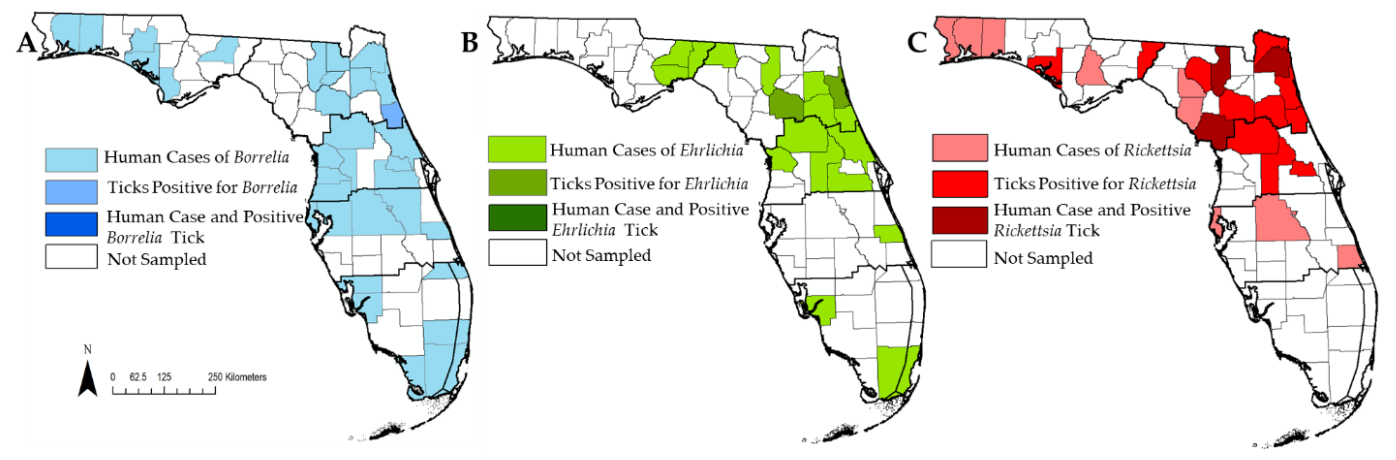

Figure 4. Maps of counties with reported human cases, tick detected with a pathogen and overlap between human cases and ticks infected with a pathogen. Thick black lines denote climatic regions. (A) Map of human cases of Lyme disease and detection of ticks with B. burgdorferi. (B) Map of human cases of Ehrlichiosis and detections of ticks infected with E. ewingii. (C) Map of human cases of Ehrlichiosis and detections of ticks infected with pathogenic Rickettsia species.

\section{Discussion}

The purpose of our investigation was to examine the distribution of tick-borne bacterial pathogens across the climatic regions of mainland Florida. We found four tick species and six bacterial species in $4 / 6$ climatic regions. All the tick and pathogen species we detected had previously been reported in Florida but our survey provided information on distribution of ticks and bacterial pathogens within a larger geographic context $[6,15-17,43]$. 
In our survey, Amblyomma americanum was the most abundant tick sampled in Florida from which multiple bacterial pathogens were detected, which is consistent with tick surveys across the Southeastern U.S. [43]. Rickettsia amblyommatis was the most common bacterial species detected in our study and is a highly prevalent bacteria across the Southeastern U.S. [3,4,6,29]. Rickettsia amblyommatis has been reported as a potentially pathogenic Rickettsia with mild symptoms [29,30]. In the U.S. there has been a decrease in hospitalizations and fatalities for RMSF cases but an increase in mild cases [30]. This trend is thought be associated with spread of $A$. americanum vectoring $R$. amblyommatis and A. maculatum vectoring $R$. parkeri, which both cause milder illness than RMSF. Further complicating the epidemiology, the causative agent of spotted fever rickettsiosis in the U.S. is either not identified or diagnostic tests used to test for RMSF cross react with other spotted fever Rickettsia species [44,45]]. Thus, the clinical and epidemiological picture of rickettsiosis remains unclear. Florida has roughly a dozen reported cases of spotted fever rickettsiosis each year, but diagnostic testing is rarely performed, which causes variation year by year if cases are reported as confirmed or probable [19]. The true infectivity of $R$. amblyommatis and R. parkeri could be underreported in the state given the mild symptoms of and imprecise diagnostic tools for these infections.

Amblyomma maculatum is considered the primary vector of R. parkeri [46]; however, our survey did not detect $R$. parkeri in this tick but instead we detected $R$. andeanae. R. andeanae has been identified as a spotted fever Rickettsia, but its pathogenicity is still unknown [47]. Populations of A. maculatum infected with R. andeanae vary across the Midwestern and Southeastern U.S. In Oklahoma and Kansas, A. maculatum infected with $R$. andeanae had a prevalence of $47-80 \%$ [48], but in southeastern states, including Florida, prevalence of $R$. andeanae is $<5 \%$ [48]. Previous investigations have found that hard ticks cannot maintain dual infections of Rickettsia by vertical transmission, which is referred to as rickettsial interference [49]. It is plausible, given that $A$. maculatum can vector $R$. parkeri and $R$. andeanae, that rickettsial interference maybe occurring and influencing the distribution of these Rickettsia species across Florida.

Dermacentor variabilis is an important vector for RMSF, which we did not detect in our study. Previous investigations have found that $R$. rickettsii is rare in D. variabilis in the U.S. [50]. We did detect $B$. lonestari and Rickettsia endosymbionts in our D. variabilis samples. Borrelia species have been previously detected in D. variabilis such as "Candidatus Borrelia texasensis" [51,52], which has only been detected in Webb County, Texas [51,52]. Dermacentor variabilis has also been shown to have bacterial species found in A. americanum including E. chaffeensis and E. ewingii, although we did not detect these pathogens in $D$. variabilis in this study [52].

Ixodes scapularis is a highly competent vector for multiple bacterial pathogens, and its role as a disease vector is poorly understood in Florida. In our investigation we only detected one I. scapularis with B. burgdorferi; however, our survey methods of I. scapularis relied solely on flagging methods and likely underestimated the distribution of low-prevalence pathogens like Borrelia in Florida. Differences in behavior between I. scapularis in the north and the southeast may have influenced our sample sizes and the ability to detect the bacteria. Because I. scapularis in the Northeastern U.S. quest above or on top of leaf litter and I. scapularis in the Southeastern U.S. quest under the leaf litter, flagging may not efficiently capture this tick species in Florida [53]. Nonetheless, flagging mimics human activity and is a good indicator of human encounters with different tick species and the pathogens to which they may be exposed [54].

In our survey we found that the temperate climatic regions (regions 1-3), which are similar in climate to the rest of the Southeastern United States [14], also had a similar tick-borne pathogen community to other southeastern regions $[8,21,22]$. In the subtropical regions of Southern Florida (climate regions 4-6), we found fewer ticks and fewer infected ticks compared to northern climate regions in Florida. Subtropical environments typically have higher amounts of precipitation during the summer months, which can influence the presence and survival of ticks [32]. This finding could also be a product of sampling bias. Dragging instead of flagging could be more appropriate for collecting ticks in subtropical parts of Florida because it may sample ticks that are more active in the leaf litter in hotter, more humid climates [53,54]. Previous studies have successfully collected ticks in Southern Florida with dragging and by surveying mammalian hosts, including deer and wild pigs $[15,16,55]$. 
Human cases of tick-borne pathogens are also present in the subtropical regions of Florida, suggesting that humans and ticks are coming in to contact even if they are not detected by flagging.

In addition to our tick and pathogen surveillance, we wanted to qualitatively assess if the distribution of infected ticks collected by flagging was similar to human case data. Visually comparing maps of both datasets, we did not find substantial overlap at the county scale with human cases and ticks infected with pathogens (Figure 4). However, our datasets only compared data collected in 2016-2017; in previous years human cases of Ehrlichiosis had been reported in counties where we found ticks to be positive for pathogens [19]. Even though we did not see direct pathogen and human case overlap, comparing both epidemiological and acarological data can still provide valuable insight by providing different perspectives on disease risk [13]. Epidemiological data alone can skew where cases are located, since the location of human case does not always correspond to where an infection was acquired and can inflate area of potential risk. However, epidemiological data do provide evidence that humans are encountering etiological disease agents. Acarological data provide information on vector distribution, abundance and pathogen prevalence. Previous investigations combining both types of data have been used to develop risk maps for Lyme disease in California, which found that areas with high tick abundance were associated with areas with high human case incidence at the subcounty level [13].

Future studies of tick pathogen surveillance in Florida should continue to incorporate the use of epidemiological and acarological data but consider differences in scale of either surveillance effort [13]. In our investigation we looked at bacterial pathogens at the scale of climatic regions. We did find overlap between infected ticks and human cases at this larger spatial scale; however, we did not see overlap of human cases and bacterial pathogens at the small county-level scale. In the California Lyme disease risk study, they found that habitats differed at the sub-county level, which influenced tick distribution [13]. Further tick bacterial pathogen surveillance in Florida should consider finer scale surveillance to account for differences in habitat types.

Lastly, we examined the seasonal patterns of ticks and R. amblyommatis. We found that $D$. variabilis and A. americanum were most active during the spring and summer months, while adult I. scapularis were active during the winter. Since, Rickettsia amblyommatis was the most abundant pathogen detected that we examined seasonality, which peaked during the spring and summer months (Figure 3). Epidemiological data for tick-borne diseases report that most human cases occur throughout the spring and summer months, which corresponds with our pathogen survey data (Tables 3 and 4) [19]. Future pathogen prevalence studies should aim to collect ticks during spring and summer months when ticks and pathogens are active and pose the greatest risk to human health.

In our investigation we were not able to address the prevalence of tick-borne bacterial pathogens. Our sampling methodology focused on collecting ticks from numerous field sites in order to provide a state-wide overview of pathogen presence based on CDC guidelines, but not estimates of prevalence. Previous studies in Florida have reported low prevalence $(0-5 \%)$ of numerous tick bacterial pathogens $[6,7,17,18,20]$. In order to estimate the prevalence of these pathogens in future studies in large tick populations with a confidence level of 0.95 , a sample size of $\sim 300$ ticks per site would be needed to accurately estimate a pathogen with the true prevalence of $1 \%[56]$.

\section{Conclusions}

Our study has provided an initial baseline for tick pathogen distribution for the state of Florida. We found multiple medically important tick species and tick-borne bacterial pathogens. Ticks and pathogens were concentrated within climatic regions 1-3 and were limited in distribution in the southern regions of the state [19]. We did not find overlap between human cases and pathogen surveillance at the county level but did at the climatic region level. Future surveillance efforts should account for scale in order to account for habitat variation within a county. Given the diversity of ticks and tick-borne bacterial pathogens in Florida, further investigations should be conducted to identify regional hotspots of tick-borne pathogens in order to fully determine the risk to public health. 
Author Contributions: C.E.D.J. contributed to the manuscript by conducting lab work, analyzing data, and writing the original draft of the manuscript. C.G. contributed by collecting field data, identifying ticks, assisting with lab protocols and providing manuscript edits. W.H.K. contributed by establishing tick collection and identification methods and assisted with manuscript edits. Z.S.W. contributed by establishing lab protocols and conducting lab work for DNA extractions. C.R.B. assisted with running lab assays and manuscript edits. G.E.G. contributed by designing the study, collecting tick samples, manuscript editing and securing research funding. S.M.W. contributed by supervising all lab activities, all manuscript editing steps and securing research funding.

Funding: This study was funded by the Centers for Disease Control and Prevention, Cooperative Agreement Number 1U01CK000510-01.

Acknowledgments: The authors thank K. Sayler for establishing biosample collection protocols. The manuscript contents are solely the responsibility of the authors and do not necessarily represent the official views of the Centers for Disease Control and Prevention.

Conflicts of Interest: The authors declare no conflict of interest and the funders had no role in the design of the study; in the collection, analyses, or interpretation of data; in the writing of the manuscript, or in the decision to publish the results.

\section{References}

1. Rosenberg, R.; Lindsey, N.P.; Fischer, M.; Gregory, C.J.; Hinckley, A.F.; Mead, P.S.; Paz-Bailey, G.; Waterman, S.H.; Drexler, N.A.; Kersh, G.J. Vital signs: Trends in reported vector-borne disease cases-United States and Territories, 2004-2016. Morb. Mortal. Wkly. Rep. 2018, 67, 496. [CrossRef] [PubMed]

2. Biggs, H.M.; Barton Behravesh, C.; Bradley, K.K.; Dahlgren, F.S.; Drexler, N.A.; Dumler, J.S.; Folk, S.M.; Kato, C.Y.L.; Ryan, R.; Levin, M.L. Diagnosis and management of tickborne rickettsial diseases: Rocky Mountain spotted fever and other spotted fever group rickettsioses, ehrlichioses, and anaplasmosis-United States: A practical guide for health care and public health professionals. MMWR Recomm. Rep. 2016, 65, 1-44. [CrossRef] [PubMed]

3. Trout Fryxell, R.T.; Hendricks, B.M.; Pompo, K.; Mays, S.E.; Paulsen, D.J.; Operario, D.J.; Houston, A.E. Investigating the adult ixodid tick populations and their associated Anaplasma, Ehrlichia, and Rickettsia bacteria at a Rocky Mountain spotted fever hotspot in Western Tennessee. Vector-Borne Zoonotic Dis. 2017, 17, 527-538. [CrossRef] [PubMed]

4. Trout Fryxell, R.; Steelman, C.; Szalanski, A.; Billingsley, P.; Williamson, P. Molecular detection of Rickettsia species within ticks (Acari: Ixodidae) collected from Arkansas United States. J. Med. Entomol. 2015, 52, 500-508. [CrossRef] [PubMed]

5. $\quad$ Fritzen, C.M.; Huang, J.; Westby, K.; Freye, J.D.; Dunlap, B.; Yabsley, M.J.; Schardein, M.; Dunn, J.R.; Jones, T.F.; Moncayo, A.C. Infection prevalences of common tick-borne pathogens in adult lone star ticks (Amblyomma americanum) and American dog ticks (Dermacentor variabilis) in Kentucky. Am. J. Trop. Med. Hyg. 2011, 85, 718-723. [CrossRef] [PubMed]

6. Mixson, T.R.; Campbell, S.R.; Gill, J.S.; Ginsberg, H.S.; Reichard, M.V.; Schulze, T.L.; Dasch, G.A. Prevalence of Ehrlichia, Borrelia, and Rickettsial agents in Amblyomma americanum (Acari: Ixodidae) collected from nine states. J. Med. Entomol. 2006, 43, 1261-1268. [CrossRef]

7. Roellig, D.M.; Fang, Q.Q. Detection of Anaplasma phagocytophilum in ixodid ticks from equine-inhabited sites in the Southeastern United States. Vector-Borne Zoonotic Dis. 2012, 12, 330-332. [CrossRef]

8. Loftis, A.D.; Mixson, T.R.; Stromdahl, E.Y.; Yabsley, M.J.; Garrison, L.E.; Williamson, P.C.; Fitak, R.R.; Fuerst, P.A.; Kelly, D.J.; Blount, K.W. Geographic distribution and genetic diversity of the Ehrlichia sp. from Panola Mountain in Amblyomma americanum. BMC Infect. Dis. 2008, 8, 54. [CrossRef]

9. Anderson, B.; Dawson, J.; Jones, D.; Wilson, K. Ehrlichia chaffeensis, a new species associated with human ehrlichiosis. J. Clin. Microbiol. 1991, 29, 2838-2842.

10. Buller, R.S.; Arens, M.; Hmiel, S.P.; Paddock, C.D.; Sumner, J.W.; Rikihisa, Y.; Unver, A.; Gaudreault-Keener, M.; Manian, F.A.; Liddell, A.M. Ehrlichia ewingii, a newly recognized agent of human ehrlichiosis. N. Engl. J. Med. 1999, 341, 148-155. [CrossRef]

11. Paddock, C.D.; Sumner, J.W.; Comer, J.A.; Zaki, S.R.; Goldsmith, C.S.; Goddard, J.; McLellan, S.L.; Tamminga, C.L.; Ohl, C.A. Rickettsia parkeri: A newly recognized cause of spotted fever rickettsiosis in the United States. Clin. Infect. Dis. 2004, 38, 805-811. [CrossRef] [PubMed]

12. Centers for Disease Control and Prevention. Available online: https://www.cdc.gov/ticks/surveillance/index. html (accessed on 29 July 2019). 
13. Eisen, R.J.; Eisen, L. Spatial modeling of human risk of exposure to vector-borne pathogens based on epidemiological versus arthropod vector data. J. Med. Entomol. 2014, 45, 181-192. [CrossRef]

14. NOAA National Centers for Environmental Information. Available online: https://www.ncdc.noaa.gov/ monitoring-references/maps/us-climate-divisions.php (accessed on 20 June 2019).

15. Allan, S.A.; Simmons, L.; Burridge, M.J. Ixodid ticks on white-tailed deer and feral swine in Florida. J. Vector Ecol. 2001, 26, 93-102. [PubMed]

16. Rogers, A.J. A Study of the Ixodid Ticks of Northern Florida, Including the Biology and Life History of Ixodes Scapularis Say (Ixodidae: Acarina). Ph.D. Thesis, University of Maryland, College Park, MD, USA, 1953.

17. Sayler, K.; Rowland, J.; Boyce, C.; Weeks, E. Borrelia burgdorferi DNA absent, multiple Rickettsia spp. DNA present in ticks collected from a teaching forest in North Central Florida. Ticks Tick-Borne Dis. 2017, 8, 53-59. [CrossRef] [PubMed]

18. Sayler, K.A.; Loftis, A.D.; Beatty, S.K.; Boyce, C.L.; Garrison, E.; Clemons, B.; Cunningham, M.; Alleman, A.R.; Barbet, A.F. Prevalence of tick-borne pathogens in host-seeking Amblyomma Am. (Acari: Ixodidae) and Odocoileus virginianus (Artiodactyla: Cervidae) in Florida. J. Med. Entomol. 2016, 53, 949-956. [CrossRef] [PubMed]

19. Florida Department of Health. Annual Morbidity Statistics Report; Technical Report for Florida Department of Health; Florida Department of Health: Tallahassee, FL, USA, 2011-2017.

20. Clark, K. Borrelia species in host-seeking ticks and small mammals in northern Florida. J. Clin. Microbiol. 2004, 42, 5076-5086. [CrossRef]

21. Burkot, T.R.; Mullen, G.R.; Anderson, R.; Schneider, B.S.; Happ, C.M.; Zeidner, N.S. Borrelia lonestari DNA in adult Amblyomma americanum ticks, Alabama. Emerg. Infect. Dis. 2001, 7, 471-473. [CrossRef]

22. Yabsley, M.J.; Dugan, V.G.; Stallknecht, D.E.; Little, S.E.; Lockhart, J.M.; Dawson, J.E.; Davidson, W.R. Evaluation of a prototype Ehrlichia chaffeensis surveillance system using white-tailed deer (Odocoileus virginianus) as natural sentinels. Vector-Borne Zoonotic Dis. 2003, 3, 195-207. [CrossRef]

23. Comer, J.A.; Nicholson, W.L.; Paddock, C.D.; Sumner, J.W.; Childs, J.E. Detection of antibodies reactive with Ehrlichia chaffeensis in the raccoon. J. Wildl. Dis. 2000, 36, 705-712. [CrossRef]

24. Castellaw, A.H.; Chenney, E.F.; Varela-Stokes, A.S. Tick-borne disease agents in various wildlife from Mississippi. Vector-Borne Zoonotic Dis. 2011, 11, 439-442. [CrossRef]

25. Demma, L.J.; Traeger, M.S.; Nicholson, W.L.; Paddock, C.D.; Blau, D.M.; Eremeeva, M.E.; Dasch, G.A.; Levin, M.L.; Singleton, J., Jr.; Zaki, S.R. Rocky Mountain spotted fever from an unexpected tick vector in Arizona. N. Engl. J. Med. 2005, 353, 587-594. [CrossRef] [PubMed]

26. Berrada, Z.L.; Goethert, H.K.; Cunningham, J.; Telford III, S.R. Rickettsia rickettsii (Rickettsiales: Rickettsiaceae) in Amblyomma americanum (Acari: Ixodidae) from Kansas. J. Med. Entomol. 2011, 48, 461-467. [CrossRef] [PubMed]

27. Burroughs, J.E.; Thomasson, J.A.; Marsella, R.; Greiner, E.C.; Allan, S.A. Ticks associated with domestic dogs and cats in Florida, USA. Exp. Appl. Acarol. 2016, 69, 87-95. [CrossRef] [PubMed]

28. Cohen, S.B.; Yabsley, M.J.; Garrison, L.E.; Freye, J.D.; Dunlap, B.G.; Dunn, J.R.; Mead, D.G.; Jones, T.F.; Moncayo, A.C. Rickettsia parkeri in Amblyomma americanum ticks, Tennessee and Georgia, USA. Emerg. Infect. Dis. 2009, 15, 1471-1473. [CrossRef] [PubMed]

29. Apperson, C.S.; Engber, B.; Nicholson, W.L.; Mead, D.G.; Engel, J.; Yabsley, M.J.; Dail, K.; Johnson, J.; Watson, D.W. Tick-borne diseases in North Carolina: Is "Rickettsia amblyommii" a possible cause of rickettsiosis reported as Rocky Mountain spotted fever? Vector-Borne Zoonotic Dis. 2008, 8, 597-606. [CrossRef] [PubMed]

30. Dahlgren, F.S.; Paddock, C.D.; Springer, Y.P.; Eisen, R.J.; Behravesh, C.B. Expanding range of Amblyomma americanum and simultaneous changes in the epidemiology of spotted fever group rickettsiosis in the United States. Am. J. Trop. Med. Hyg. 2016, 94, 35-42. [CrossRef] [PubMed]

31. Glass, G.E.; Ganser, C.; Kessler, W.H. Standardized Ixodid Tick Surveys in Mainland Florida. Insects 2019, 10, 235. [CrossRef]

32. Kessler, W.H.; Blackburn, J.K.; Sayler, K.A.; Glass, G.E. Estimating the geographic distribution of host-seeking adult Amblyomma americanum (Acari: Ixodidae) in Florida. J. Med. Entomol. 2019, 56, 55-64. [CrossRef]

33. Clifford, C.M.; Anastos, G.; Van der Borght-Elbl, A. The Larval Ixodid Ticks of the Eastern United States (Acarina-Ixodidae); Entomological Society of America: Annapolis, MD, USA, 1961; Volume 26, pp. 435-448.

34. Keirans, J.E.; Litwak, T.R. Pictorial Key to the Adults of Hard Ticks, Family Ixodidae (Ixodida, Ixodoidea), East of the Mississippi River. J. Med. Entomol. 1989, 26, 435-448. [CrossRef] 
35. Durden, L.A.; Keirans, J.E. Nymphs of the Genus Ixodes (Acari: Ixodidae) of the United States: Taxonomy, Identification Key, Distribution, Hosts, and Medical/Veterinary Importance; Entomological Society of America: Lanham, MD, USA, 1996; p. 95.

36. Keirans, J.E.; Durden, L.A. Illustrated key to nymphs of the tick genus Amblyomma (Acari: Ixodidae) found in the United States. J. Med. Entomol. 1998, 35, 489-495. [CrossRef]

37. Qiagen DNeasy Blood and Tissue Handbook. Available online: https://www.qiagen.com/us/resources/ resourcedetail?id=6b09dfb8-6319-464d-996c-79e8c7045a50\&lang=en (accessed on 28 July 2019).

38. Thermo Fisher Trizol Reagent Hanbook. Available online: http://tools.thermofisher.com/content/sfs/manuals/ trizol_reagent.pdf (accessed on 28 July 2019).

39. R Core Team. R: A Language and Environment for Statistical Computing; R Foundation for Statistical Computing: Vienna, Austria, 2013.

40. Tabara, K.; Arai, S.; Kawabuchi, T.; Itagaki, A.; Ishihara, C.; Satoh, H.; Okabe, N.; Tsuji, M. Molecular survey of Babesia microti, Ehrlichia species and Candidatus Neoehrlichia mikurensis in wild rodents from Shimane Prefecture, Japan. Microbiol. Immunol. 2007, 51, 359-367. [CrossRef] [PubMed]

41. Barbour, A.G.; Maupin, G.O.; Teltow, G.J.; Carter, C.J.; Piesman, J. Identification of an uncultivable Borrelia species in the hard tick Amblyomma americanum: Possible agent of a Lyme disease-like illness. J. Infect. Dis. 1996, 173, 403-409. [CrossRef] [PubMed]

42. Regnery, R.L.; Spruill, C.L.; Plikaytis, B.D. Genotypic Identification of Rickettsiae and Estimation of Intraspecies Sequence Divergence for Portions of 2 Rickettsial Genes. J. Bacteriol. 1991, 173, 1576-1589. [CrossRef] [PubMed]

43. Goddard, J.; Sumner, J.; Nicholson, W.; Paddock, C.; Shen, J.; Piesman, J. Survey of ticks collected in Mississippi for Rickettsia, Ehrlichia, and Borrelia species. J. Vector Ecol. 2003, 28, 184-189. [PubMed]

44. Eremeeva, M.; Yu, X.J.; Raoult, D. Differentiation among Spotted-Fever Group Rickettsiae Species by Analysis of Restriction-Fragment-Length-Polymorphism of PCR-Amplified Dna. J. Clin. Microbiol. 1994, 32, 803-810. [PubMed]

45. Raoult, D.; Parola, P. Rocky Mountain spotted fever in the USA: A benign disease or a common diagnostic error? Lancet Infect. Dis. 2008, 8, 587-589. [CrossRef]

46. Paddock, C.D.; Fournier, P.; Sumner, J.W.; Goddard, J.; Elshenawy, Y.; Metcalfe, M.G.; Loftis, A.D.; Varela-Stokes, A. Isolation of Rickettsia parkeri and Identification of a Novel Spotted Fever Group Rickettsia sp. from Gulf Coast Ticks (Amblyomma maculatum) in the United States. Appl. Environ. Microbiol. 2010, 76, 2689-2696. [CrossRef] [PubMed]

47. Paddock, C.D.; Denison, A.M.; Dryden, M.W.; Noden, B.H.; Lash, R.R.; Abdelghani, S.S.; Evans, A.E.; Kelly, A.R.; Hecht, J.A.; Karpathy, S.E.; et al. High prevalence of "Candidatus Rickettsia andeanae" and apparent exclusion of Rickettsia parkeri in adult Amblyomma maculatum (Acari: Ixodidae) from Kansas and Oklahoma. Ticks Tick-Borne Dis. 2015, 6, 297-302. [CrossRef] [PubMed]

48. Jiang, J.; Stromdahl, E.Y.; Richards, A.L. Detection of Rickettsia parkeri and Candidatus Rickettsia andeanae in Amblyomma maculatum Gulf Coast Ticks Collected from Humans in the United States. Vector-Borne Zoonotic Dis. 2012, 12, 175-182. [CrossRef] [PubMed]

49. Burgdorfer, W.; Hayes, S.F.; Mavros, A.J. Nonpathogenic rickettsiae in Dermacentor andersoni: A limiting factor for the distribution of Rickettsia rickettsii. Rickettsiae and rickettsial diseases. In Rickettsiae and Rickettsial Diseases; Burgdorfer, W., Anacker, R.L., Eds.; Academic Press: New York, NY, USA, 1980; pp. 585-594.

50. Stromdahl, E.Y.; Jiang, J.; Vince, M.; Richards, A.L. Infrequency of Rickettsia rickettsii in Dermacentor variabilis Removed from Humans, with Comments on the Role of Other Human-Biting Ticks Associated with Spotted Fever Group Rickettsiae in the United States. Vector-Borne Zoonotic Dis. 2011, 11, 969-977. [CrossRef]

51. Lin, T.; Gao, L.H.; Seyfang, A.; Oliver, J.H. 'Candidatus Borrelia texasensis', from the American dog tick Dermacentor variabilis. Int. J. Syst. Evol. Microbiol. 2005, 55, 685-693. [CrossRef] [PubMed]

52. Williamson, P.C.; Billingsley, P.M.; Teltow, G.J.; Seals, J.P.; Turnbough, M.A.; Atkinson, S.F. Borrelia, Ehrlichia, and Rickettsia spp. in Ticks Removed from Persons, Texas, USA. Emerg. Infect. Dis. 2010, 16, 441-446. [CrossRef] [PubMed]

53. Arsnoe, I.M.; Hickling, G.J.; Ginsberg, H.S.; McElreath, R.; Tsao, J.I. Different Populations of Blacklegged Tick Nymphs Exhibit Differences in Questing Behavior That Have Implications for Human Lyme Disease Risk. PLoS ONE 2015, 10, e0127450. [CrossRef] [PubMed] 
54. Ginsberg, H.S.; Ewing, C.P. Comparison of flagging, walking, trapping, and collecting from hosts as sampling methods for northern deer ticks, Ixodes dammini, and lone-star ticks, Amblyomma americanum (Acari: Ixodidae). Exp. Appl. Acarol. 1989, 7, 313-322. [CrossRef] [PubMed]

55. Merrill, M.M.; Boughton, R.K.; Lord, C.C.; Sayler, K.A.; Wight, B.; Anderson, W.M.; Wisely, S.M. Wild pigs as sentinels for hard ticks: A case study from south-central Florida. Int. J. Parasitol. Parasites Wildl. 2018, 7, 161-170. [CrossRef] [PubMed]

56. Sergeant, ESG. Epitools Epidemiological Calculators. Ausvet Pty Ltd. Available online: http://epitools. ausvet.com.au (accessed on 18 December 2018).

(C) 2019 by the authors. Licensee MDPI, Basel, Switzerland. This article is an open access article distributed under the terms and conditions of the Creative Commons Attribution (CC BY) license (http://creativecommons.org/licenses/by/4.0/). 